\author{
M. ${ }^{a}$ del Carmen Pedraza Castañeda ${ }^{1, *}$ \\ Guillermina Pérez Jiménez ${ }^{1}$ \\ Laura Solís Flores² \\ J. Eduardo Barrera Arenas ${ }^{1}$ \\ Pablo Hernández Jiménez ${ }^{3}$ \\ M. ${ }^{a}$ Virginia Xochigua Angulo ${ }^{4}$ \\ Guillermo Meléndez Mier ${ }^{5}$
}

1. Maestra en Terapia de Heridas, Estomas y Quemaduras. Hospital General de México "Dr. Eduardo Liceaga". Ciudad de México. México.

2. Maestra en Investigación de los Servicios de Salud. Hospital General de México "Dr. Eduardo Liceaga". Ciudad de México. México.

3. Pasante de Enfermería. Hospital General de México "Dr. Eduardo Liceaga". Ciudad de México. México.

4. Enfermera General. Hospital General de México "Dr. Eduardo Liceaga". Ciudad de México. México.

5. Subdirector de Investigación. Universidad de la Salud. Ciudad de México. México.

*Autor para correspondencia.

Correo electrónico: carmenpedraza@hotmail.com; investigalaura4@yahoo.com

(M. ${ }^{\text {a }}$ del Carmen Pedraza Castañeda).

Recibido el 31 de agosto de 2020; aceptado el 4 de enero de 2021

\section{Coste-efectividad de la terapia avanzada con productos de cura en ambiente húmedo comparada con terapia tradicional en el tratamiento de úlceras por presión de categorías I y II}

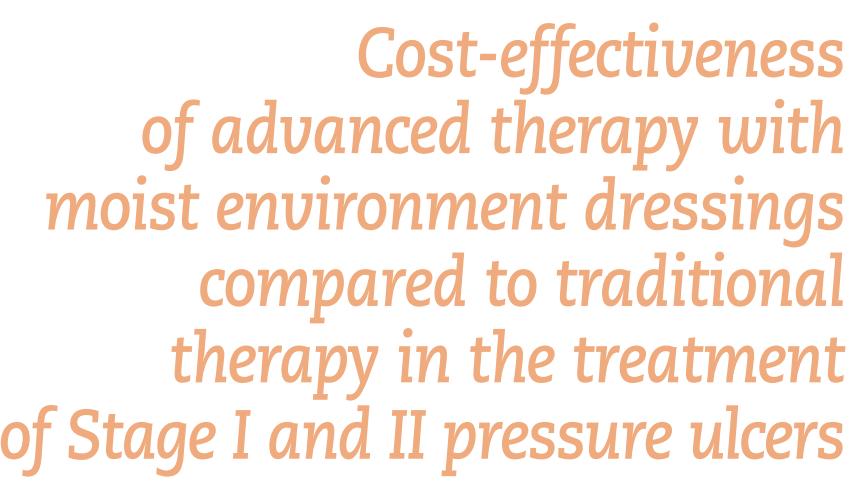
moist environment dressings compared to traditional therapy in the treatment of Stage I and II pressure ulcers

\section{RESUMEN}

Objetivo: Demostrar el coste-efectividad de la cura en ambiente húmedo (CAH) sobre la cura tradicional (CT) en pacientes hospitalizados con úlceras por presión (UPP) de categorías I y II. Metodología: Estudio longitudinal comparativo, aleatorizado, abierto, dividido en dos grupos, tratados y seguidos durante 4 semanas. El tamańo de la muestra se calculó con base en el metaanálisis de Heyer. Creamos un árbol de decisión para comparar el coste-efectividad de cada cura. Los datos se recopilaron directamente de los formularios de informes de casos de pacientes y los costes se extrajeron de los registros hospitalarios. Realizamos análisis de sensibilidad y costeefectividad incrementales. Resultados: El análisis mostró un dominio de

la CAH sobre la CT $(223,00 € / 0,84$ vs. $298,00 € / 0,51)$, ya que fue más rentable al final del estudio. La hospitalización fue más corta en la CAH y la curación se logró en menos días que con la CT, lo que tiene un impacto en los costes relacionados con los tiempos de atención de enfermería, gasto de recursos materiales y días de hospitalización de los pacientes. Conclusiones:

La CAH es más coste-efectiva que la CT cuando se aplica a pacientes hospitalizados con UPP de categorías I y II.

PALABRAS CLAVE: Coste-efectividad, úlcera por presión, análisis de coste, enfermería.

\section{ABSTRACT}

Objective: To demonstrate the cost-effectiveness of the moist environment dressings treatment over the Traditional Cure (TC), in hospitalized patients, with Pressure Ulcers (UPP) categories I and II. Methods: This is a comparative, randomized, open-label, longitudinal study, comprising 60 patients, divided into two groups, treated and followed for four weeks. The sample size was calculated based upon the meta-analysis by Heyer. We built a decision tree to compare the cost-effectiveness of each therapy. Data were collected directly from patients' case report forms and costs extracted from hospital records. Additionally, we performed sensitivity and incremental cost-effectiveness analyses. Results: The analysis showed a dominance of advanced therapy over traditional therapy (€ 223.00/0.84 vs. $€ 298.00 / 0.51$ ), as it was more cost-effective at the end of the study. Hospitalization was shorter in the advanced therapy and healing was achieved in fewer days than the traditional therapy. Conclusions: Advanced therapy with healing products in a humid environment is more cost-effective than traditional therapy when applied to hospitalized patients with category I and II pressure ulcers.

KEYWORDS: Cost-efectiveness, pressure ulcers, cost analysis, nursing.

\section{У INTRODUCCIÓN}

El coste-efectividad se evalúa mediante el análisis que relaciona los costes de los diversos métodos formales para evaluar la rentabilidad entre diferentes intervenciones, una intervención o tratamiento con los resultados obtenidos. La rentabilidad es relativa: una intervención que aparentemente es cara puede ser rentable en comparación con una alternativa más barata si los beneficios obtenidos son mayores. En general, se produce un mayor coste para obtener un mayor beneficio. Dentro de las ventajas principales de aplicar cura en ambiente húmedo $(\mathrm{CAH})$ se 
encuentran: mayor estimulación de la cicatrización, menor dolor, control del exudado y mayor efectividad clínica y rentabilidad que la cura tradicional (CT). Los costes económicos asociados con las úlceras por presión (UPP) son notablemente altos, a lo que es necesario agregar el impacto emocional y el sufrimiento (es decir, costes intangibles) ${ }^{1}$.

Por otra parte, en los resultados de un metaanálisis con los estudios de muestra suficiente $(\mathrm{N}>50)$ se comparan productos de tratamiento en ambiente húmedo con tratamiento tradicional, apreciándose claramente una mayor efectividad clínica de la $\mathrm{CAH}(63,51 \%$ de cicatrizaciones) frente a la cura seca tradicional (36,96\% de cicatrizaciones) ${ }^{2}$.

El término "coste-efectividad" significa que algo es "efectivo o productivo en relación con su coste". En la atención sanitaria, una intervención coste-efectiva proporciona beneficios clínicos a un coste razonable y los beneficios obtenidos superan a los que se obtendrían si los recursos se utilizaran en otra cosa.

Los costes se pueden dividir en:

1. Costes directos: son aquellos en los que incurre el sistema sanitario y/o el paciente como resultado directo de la enfermedad, por ejemplo, una herida, y de su tratamiento asociado.

2. Costes indirectos: no son tan evidentes; incluyen las pérdidas para la sociedad provocadas por la enfermedad y su tratamiento ${ }^{3}$.

Además, las heridas crónicas suponen un problema asistencial muy importante para el sistema sanitario y un elevado coste económico debido al aumento de las estancias hospitalarias y a necesidades de cuidados prolongados ${ }^{4}$. Por lo tanto, cada uno de los materiales debe ponerse a prueba para medir su efectividad en la atención de los pacientes de acuerdo con el tipo de herida, el tiempo de las curaciones y los recursos necesarios para el logro de su cicatrización ${ }^{5}$.

Los resultados de una evaluación económica pueden representarse gráficamente a través del plano de coste-efectividad, el cual expresa, en el eje de abscisas, el efecto en salud, y en el eje de ordenadas, el coste asociado. Si se asume que la CT se encuentra en el origen, al realizar una comparación con una nueva intervención se generan cuatro posibles situaciones:

1. Que la nueva terapia sea más efectiva y menos costosa.

2. Que la nueva terapia sea más efectiva pero más costosa.

3. Que la nueva terapia sea menos efectiva pero menos costosa.

4. Que la nueva terapia sea menos efectiva y más costosa ${ }^{6}$.

El análisis de coste-efectividad (ACE) es la técnica de evaluación más utilizada en farmacoeconomía para la comparación de distintas alternativas de intervención terapéutica y se encuentran entre los criterios requeridos por el NICE (National Institute for Clinical Excellence) para recomendar un tratamiento.

El ACE es una técnica de evaluación económica que trata de comparar el coste y el efecto clínico de dos o más intervenciones alternativas, potencialmente competidoras y, en general, mutuamente excluyentes, para determinar cuál de ellas es mejor desde un punto de vista económico y clínico ${ }^{7}$.

La Organización Mundial de la Salud ha tomado la presencia de UPP como un indicador de la calidad de los cuidados de enfermería. En México, la Secretaría de Salud ha dispuesto la apertura de clínicas de heridas en diferentes unidades hospitalarias en el país, en donde la atención es proporcionada por profesionales de enfermería especializados en la cura de heridas con diferentes tratamientos, entre ellos el de la $\mathrm{CAH}$, como una política de estado orientada a atender a la población con estas necesidades.

En un estudio anterior de coste-beneficio diseńado con otro tipo de $\mathrm{CAH}$ para heridas se propuso la necesidad de abrir nuevas hipótesis para estudios posteriores en la cura de heridas ${ }^{8}$. En esta investigación se obtuvo el coste-efectividad del tratamiento de $\mathrm{CAH}$ (apósitos de poliuretano, ácidos grasos hiperoxigenados y un ungüento tópico enzimático) comparado con la CT (agua y jabón) en personas con UPP de categorías I y II.

\section{Y OBJETIVO}

Demostrar el coste-efectividad de la terapia avanzada con productos de $\mathrm{CAH}$ sobre la tradicional, en pacientes hospitalizados, con UPP de categorías I y II.

\section{Variables objeto del estudio}

Edad, sexo, coste diario de tratamiento, grupo de tratamiento, días de curación, días de hospitalización, clasificación de Katz, motivo del alta hospitalaria, lesión al inicio del tratamiento, porcentaje de reducción de la lesión al final del tratamiento, clasificación de RESVECH, localización de la lesión, número de sujetos con cicatrización de la lesión antes de 20 días.

\section{Consideraciones éticas}

El estudio fue revisado y aprobado por los comités de ética en investigación y de investigación del Hospital General de México "Dr. Eduardo Liceaga” y se apegó a los principios de la declaración de Helsinki 2013, de la Ley General de Salud y el Reglamento de la Ley General de Salud en Materia de Investigación en Salud. Cada participante o su representante legal aceptaron firmar el consentimiento informado para ser incluidos en el estudio.

\section{METODOLOGÍA}

Se trata de un estudio abierto, longitudinal, paralelo, prospectivo y comparativo en 60 pacientes hospitalizados, asignados en dos grupos para cada una de las curas, con seguimiento de un mes para evitar complicaciones, a los que se proporcionó CAH o CT.

Criterios de inclusión: pacientes mayores de 18 años, hospitalizados, con UPP de categoría I y II de acuerdo con la clasificación del GNEAUPP', con un plan de hospitalización mínimo de 4 semanas y que aceptaron participar en el estudio (tabla 1).

Los investigadores aplicaron el cuestionario de actividades de la vida diaria reportado por Katz y se hizo una medición del área y la profundidad de la lesión al ingreso al estudio. La efectividad se midió con la reducción de la lesión al final del estudio y los días de hospitalización.

\section{Tamaño de la muestra}

La estimación del tamaño de muestra para este estudio se basó en los resultados de un metaanálisis de Heyer ${ }^{10}$ que describe una razón de momios de 2,57 entre las dos curas a favor de la CAH en el caso de UPP. La razón de momios se transformó a "d" de Cohen de acuerdo con la recomendación de Borenstein, considerando un valor $\alpha$ de 0,05 y un poder de la prueba de $90 \%$.

De acuerdo con estos datos, el valor identificado en la población en estudio, en 2016, las UPP tuvieron una prevalencia del 11,6\% de un total de 765 pacientes atendidos ese mismo año con distintos tipos de lesiones ${ }^{11}$, lo que permitió identificar la factibilidad de la muestra.

Se calculó el tamaño de la muestra para cada grupo en 79 sujetos, dando un total de 158 pacientes; se agregó un $20 \%$ por pérdidas de 
Tabla 1. Fases del estudio

\begin{tabular}{|c|c|c|}
\hline Fase & Cura tradicional & Cura avanzada \\
\hline 1 & \multicolumn{2}{|l|}{$\begin{array}{l}\text { - Asignación de los pacientes a los grupos de acuerdo con la tabla aleatoria } \\
\text { - Identificación de los sujetos de estudio } \\
\text { - Aplicación de consentimiento informado }\end{array}$} \\
\hline II & $\begin{array}{l}\text { a) Valoración integral con el formato de clínica de heridas con la escala } \\
\text { de Resvech } \\
\text { b) Asignación de tratamiento de acuerdo con la tabla aleatoria } \\
\text { c) Inicio de tratamiento con agua y jabón con clorhexidina, colchón de } \\
\text { presión alterna, almohadillas para liberar sitios de presión y vaselina } \\
\text { líquida } \\
\text { d) Educación para la salud (cuidados de la piel, movilización, superficies } \\
\text { especiales para el manejo de la presión, SEMP) y recomendaciones } \\
\text { de alimentación } \\
\text { e) Visitas de lunes a viernes a los pacientes }\end{array}$ & $\begin{array}{l}\text { a) Valoración integral con el formato de clínica de heridas con la escala } \\
\text { de Resvech } \\
\text { b) Asignación de tratamiento de acuerdo con la tabla aleatoria } \\
\text { c) Inicio del tratamiento con apósitos de espuma hidrocelular con } \\
\text { adhesivo de silicona, ácidos grasos hiperoxigenados y cloranfenicol } \\
\text { colagenasa (ulcoderma), SEMP, almohadillas para liberar sitios de } \\
\text { presión y vaselina líquida } \\
\text { d) Educación para la salud (cuidados de la piel, movilización, SEMP) } \\
\text { y recomendaciones de alimentación } \\
\text { e) Visitas } 2 \text { a } 3 \text { veces por semana }\end{array}$ \\
\hline III & $\begin{array}{l}\text { Seguimiento } \\
\text { a) Revaloración de lunes a viernes de la herida y de cuidados } \\
\text { recomendados } \\
\text { b) Registro de material utilizado para las curaciones } \\
\text { c) Cierre de herida o alta por cualquier motivo }\end{array}$ & $\begin{array}{l}\text { Seguimiento } \\
\text { a) Revaloración de } 2 \text { a } 3 \text { veces por semana de la herida y de cuidados } \\
\text { recomendados } \\
\text { b) Registro de material utilizado para las curaciones } \\
\text { c) Cierre de herida o alta por cualquier motivo. } 0 \text { cambio de } \\
\text { tratamiento por límite de tiempo para cierre ( } 20 \text { días) }\end{array}$ \\
\hline
\end{tabular}

pacientes en seguimiento, resultando en total 190, distribuidos en 95 pacientes por cada grupo de estudio. Se llevó a cabo una estrategia de aleatorización utilizando la aplicación randomization.com. Se programó una distribución aleatoria para la integración de los participantes a las distintas terapias.

\section{Análisis estadístico}

Para el proceso de aleatorización, se hizo la selección en bloques de 6 sujetos cada uno para mantener la independencia. El estudio fue abierto, por lo que los investigadores conocían el tratamiento asignado, pero no lo podían modificar.

Se realizó un análisis de la demografía de cada grupo, incluyendo edad, sexo, categoría de la lesión, tiempo de hospitalización y se comparó mediante prueba de la $\chi^{2}$.

Para el ACE se utilizó el programa TreeAge ${ }^{\circledast}$. Los costes se calcularon en euros para la comparación de la prueba. La efectividad se calculó en función del número de sujetos que alcanzaron diferentes grados de mejoría o que tuvieron el cierre completo de la lesión al final del estudio en cada grupo. Se tomó en cuenta el tiempo transcurrido hasta la mejoría o curación de la herida como parte de la efectividad. Se llevó a cabo un análisis de sensibilidad para evaluar el coste-efectividad entre las dos terapias y se hizo una estimación con el método Monte Carlo con 10000 replicaciones para evaluar la distribución de los datos.

Finalmente, se calculó el coste-efectividad con base en costes y en la reducción de la lesión al final de cada tratamiento, se calculó además el dominio de cada tratamiento y el coste incremental entre las dos terapias. Adicionalmente, se llevó a cabo una prueba de supervivencia tomando como punto final la cicatrización de la lesión y los días en los que ocurrió.

\section{У RESULTADOS}

Se observó una diferencia en el promedio de edad entre cada grupo (tradicional $=59,13 \pm 15,57$ vs. avanzado $=49,06 \pm 15,85$, prueba de $t$
= 0,0160); no existió diferencia en las características basales de acuerdo con la distribución de sexos mediante la prueba de la $\chi^{2}(p=0,500)$, así como tampoco en el estado civil $(p=0,492)$. En cuanto a la localización de la herida (glúteo, sacro, talón, tercio inferior de la pierna y trocánter) $(p=0,252)$ tampoco hubo diferencia.

Cabe destacar que el índice de Katz valorado al inicio del estudio fue semejante entre los dos grupos, $p=0,337$, debido a las condiciones de los pacientes hospitalizados en los que existe dependencia para realizar sus actividades básicas.

En cuanto al número de curaciones que recibieron los pacientes, según la cura que se les proporcionó, el grupo con CT necesitó mayor número de curaciones [CT 299 vs. CA 155 ( $p \leq 0,0003)$ ].

Respecto al tiempo de cicatrización de las heridas, en el grupo que recibió CAH, 22 de los pacientes lograron cerrar sus heridas en menos de 4 semanas, y en el grupo con CT solo 4 lo lograron en el mismo tiempo. La diferencia entre ambos grupos dio un valor de $p$ altamente significativo $(p=0,000)$.

El reclutamiento del estudio se suspendió al llegar a 30 pacientes en cada uno debido a la contundente diferencia en la respuesta a la $\mathrm{CAH}$ comparado con la CT. Los resultados se presentaron ante los comités de ética en investigación e investigación, quienes autorizaron suspender el reclutamiento de pacientes.

\section{У DISCUSIÓN}

La implementación de la CAH en pacientes hospitalizados con UPP, categorías I y II, promete tener un beneficio importante para las personas y el hospital.

En el ACE, el árbol de decisión mostró que la CAH fue más coste-efectiva, comparada con la tradicional, quedando la CAH como dominante sobre la tradicional.

En la figura 1 se demuestra que el coste-efectividad fue de 580,53 euros sobre la CT, que si se convierte a la prevalencia nacional del 7,6\% de los 129,2 millones de habitantes en 2017 representa 9819200 de pacientes que al ańo sufren UPP, de las cuales el $80 \%$ son de categorías I y II; 


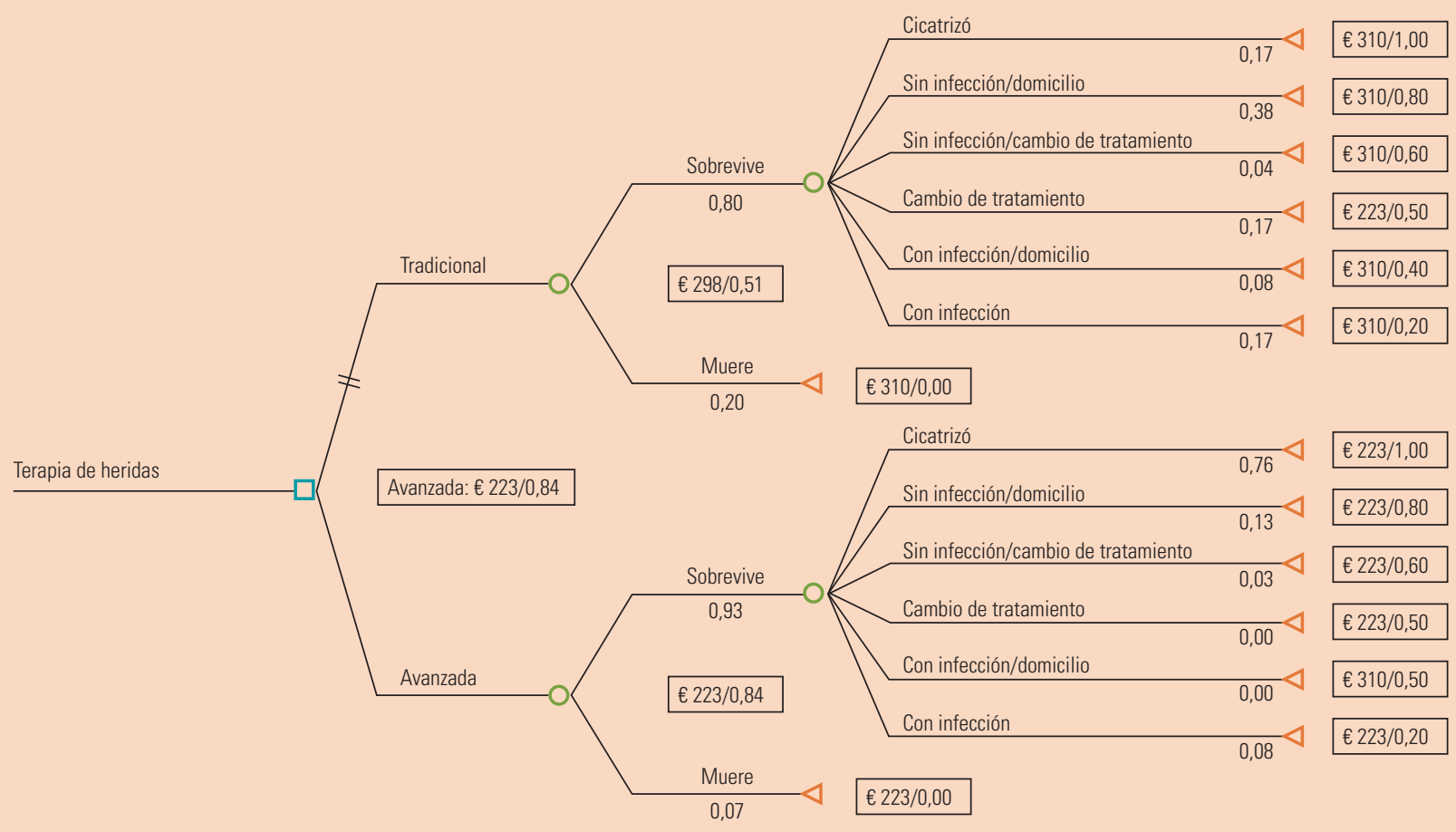

Figura 1. Árbol de decisión.

esto equivale a 7855360 pacientes, resultando que el ahorro para la sociedad mexicana por concepto de CAH para UPP es de 5436 697,5 euros, como se muestra en la tabla de coste-efectividad.

Por otra parte, la efectividad, medida en días de hospitalización y en número de pacientes que lograron la cicatrización al final del estudio, fue mayor en el grupo de terapia avanzada, lo que demuestra su dominio sobre la CT, quedando claro que es la opción más efectiva y menos costosa. La estrategia de CT es dominada por la estrategia de CAH; por lo tanto, el coste-efectividad incremental en que la CAH domina a la CT, tanto en coste como en efectividad, con un valor de 580,53 euros (tabla 2), resultado que se muestra en la gráfica de dominio, en la que se observa por cuadrantes que la CAH es más coste-efectiva que la CT, esta es más precisa en mostrar el dominio de la $\mathrm{CAH}$ (fig. 2).

Además del coste-efectividad dominado por la $\mathrm{CAH}$, el porcentaje de sujetos curados a lo largo del tiempo fue significativamente mayor con la CAH, dominando sobre la CT (fig. 3).

La técnica de evaluación económica elegida depende de la naturaleza de los beneficios que se van a estudiar. En el caso del ACE, los beneficios se expresan en términos de mejoría de las heridas correlacionadas con el tiempo y pueden ser expresados como el porcentaje de curación, debido al porcentaje de pacientes que alcanza un determinado objetivo terapéutico.

Tal como señala Zárate, dentro de las posibilidades del resultado del coste-efectividad se encontró que la CAH es más efectiva y menos costosa; con un cálculo de tratamiento durante un mes, debido a la necesidad de incrementar el total de curaciones en la CT; también los resultados de la revisión documental muestran la existencia de pruebas a favor de la mayor eficacia clínica de los apósitos basados en CAH frente a los apósitos tradicionales. Los productos de $\mathrm{CAH}$ son más rentables que el tratamiento en ambiente seco, con gasas o tradicionales. Aunque el número de estudios que analizan los aspectos económicos del tratamiento local de las UPP no son numerosos, las conclusiones que se extraen de ellos muestran claramente la ventaja de los productos de $\mathrm{CAH}$ al considerar la dimensión coste y efectividad, a pesar de que los apósitos de $\mathrm{CAH}$ tienen un mayor precio unitario, cuando se considera el coste por proceso, en el que se incluyen otros elementos asociados, número de curas, tiempo de enfermería y tiempo de hospitalización.

Los hallazgos de Reyes-Rocha también demostraron que el coste total de la CAH tendría que ser analizado en función no solo de la inversión realizada en los apósitos, sino también en función del gasto que

Tabla 2. Análisis de coste-efectividad incremental

\begin{tabular}{|l|c|c|c|c|c|c|}
\hline \multicolumn{1}{|c|}{ Estrategia } & Coste (€) & Coste incr. (€) & Efectividad & Efectividad incr. & C/E (€) & Incr. C/E (ICER) \\
\hline Avanzada & 223 & & 0,84 & & 266,73 & \\
\hline Tradicional & 298 & $€ 75$ & 0,51 & $-0,32$ & 580,53 & (Dominada) \\
\hline
\end{tabular}

Todas las opciones se refieren a una basal común. 


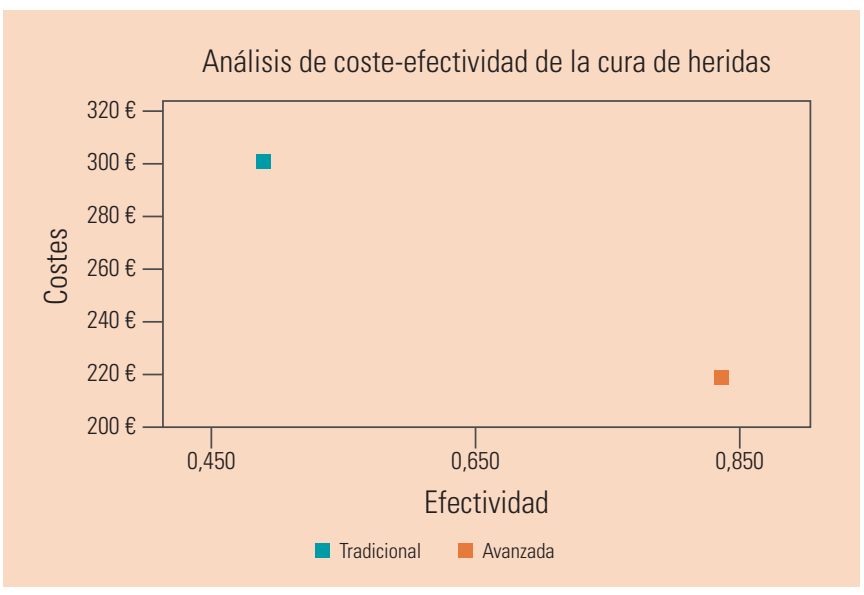

Figura 2. Gráfica de dominio de terapia avanzada.

la institución debe realizar en el pago del tiempo de enfermera en la atención del paciente y del periodo de cicatrización de la herida. Lo que comprueba este estudio.

En común con García, el coste global del tratamiento de UPP con $\mathrm{CAH}$, al igual que los productos basados en ambiente húmedo, es menor que el del tratamiento con cura en ambiente seco $^{2}$.

El coste de tratar las UPP en Espańa supone un importante desembolso para el sistema sanitario y la sociedad en general, rondando cifras que superan el 5\% del gasto sanitario anual de este país en lo siguiente: el coste medio del tiempo de enfermería empleado en el tratamiento de las UPP representa un 19,2\% de su coste total; las UPP consumen una gran cantidad de tiempo de enfermería, el coste total de la atención a las mismas está muy directamente condicionado por aquellos pacientes cuya estancia, tanto en hospitales como en centros sociosanitarios, se prolonga en el tiempo, representando este incremento de las estancias un $66 \%$ del coste total.

En relación con el coste con CT y CAH podemos destacar que: el mayor impacto del cambio de CT a CAH se centra en el tiempo de enfermería; esto es debido a una menor necesidad de cambios de cura y a un menor tiempo para cicatrizar. Por ejemplo, la cicatrización de una úlcera con apósitos de $\mathrm{CAH}$ requiere una media de 13,6 horas de enfermería menos que cuando se cura con apósitos de CT. El coste total anual estimado del tratamiento de las UPP en Espańa sería de 602 millones de euros si se tratasen todas con apósitos tradicionales frente a un coste de 461 millones de euros si fuesen tratadas con apósitos de $\mathrm{CAH}$, es decir, 141 millones o un $31 \%$ más con la CT.

En este estudio, el promedio de horas de atención en el caso de la CT va de 15 a 20 horas o más (coste aproximado 325,7 euros) y en el caso de CAH se reduce de 7 a 3 (coste aproximado de 50,4) tiempos de curación de enfermería.

El estudio de costes asociado a las UPP más preciso hasta la fecha fue el llevado a cabo por Bennet, Dealey y Posnett en el año 2002 en el Reino Unido, donde se estableció que el coste de tratar una UPP partía de 1064 libras esterlinas para el estadio I; el coste se incrementa con el estadio. Basándose en este mismo estudio, Posnett y Torra extrapolaron en 2003 los datos a la realidad espańola determinando costes por episodio de 211 euros para el estadio I. El coste del tratamiento de las UPP está directamente relacionado con su impacto como problema de salud y con el tiempo necesario para su cicatrización, además de importantes costes directos e indirectos. Entre los primeros se incluyen el tiempo de los profesionales, el coste en materiales, el aumento de la estancia hospitalaria y los costes relacionados con las complicaciones ${ }^{12}$.
Comparación de días para curación entre terapia avanzada vs. tradicional

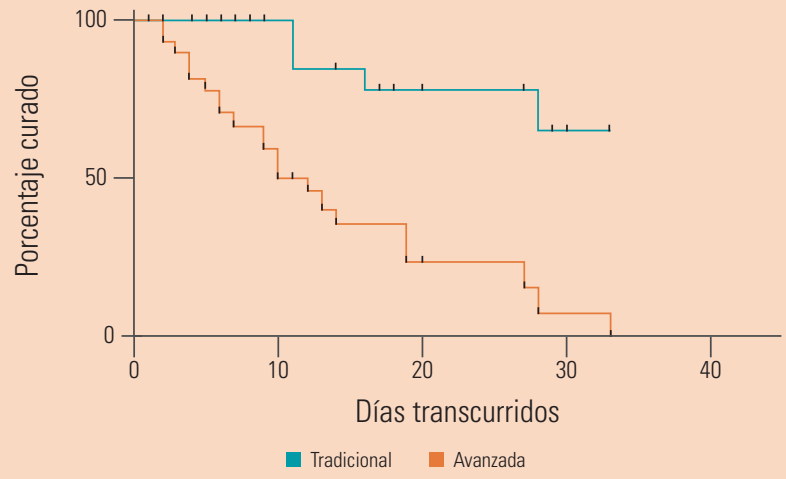

\begin{tabular}{|l|l|}
\hline Log-rank (Mantel-Cox) test & \multicolumn{1}{|l|}{} \\
\hline$\chi^{2}$ & 17,97 \\
\hline DF & 1 \\
\hline Valor de $p$ & $<0,0001$ \\
\hline
\end{tabular}$\quad$\begin{tabular}{|l|l|}
\hline Hazard ratio (log-rank) & A/B \\
\hline Ratio (y su recíproco) & 6,04 \\
\hline IC 95\% de la ratio & 2,765 a 13,2 \\
\hline
\end{tabular}

Figura 3. Tasa de supervivencia hasta la curación por grupo.

Podemos agregar que el coste por paciente debe considerar que un paciente puede tener más de una lesión. Para este estudio mexicano, se consideró la lesión con mayor extensión para su estudio y tratamiento, y fuera de estudio se proporcionó el tratamiento necesario a las demás lesiones en los casos existentes.

Soldevilla también confirma que es posible calcular de manera separada los costes por tipo de recurso, costes materiales (apósitos y antibióticos), tiempo de enfermería, días extras de estancia de hospital y centro sociosanitario $^{13}$

Así mismo, Restrepo afirma que existen implicaciones para el individuo, la familia/cuidadores y para el sistema de salud en el aumento de los costes directos e indirectos, lo que se traduce en elevados gastos en la curación, aumento de la estancia hospitalaria, tiempo de atención de los profesionales de enfermería y demandas legales, puesto que la aparición de estas lesiones determina la efectividad del cuidado de la piel ${ }^{14}$. Demostramos, con los resultados de este estudio, que la utilización de apósitos de CT representa un incremento de coste por paciente entre un $21 \%$ y un $41 \%$.

Coincidimos con Soldevilla et al., quienes señalaron que el gasto en materiales es más bajo por paciente en el caso de los apósitos de $\mathrm{CAH}$ aun cuando el precio unitario de estos es mayor ${ }^{15}$.

Se comprobó la efectividad de la CAH, por lo que podemos asegurar que la inversión que pudieran hacer los hospitales beneficiaría a los pacientes en el proceso de cicatrización de sus heridas, sobre todo cuando estas son iniciales; limitaría el daño y prevendría que aumenten los grados de complicación y/o la presencia de nuevas lesiones.

En el caso de la población en estudio, las categorías I y II se encontraron en $19,8 \%$ y $34,1 \%$, respectivamente. Estamos de acuerdo en que los pacientes deben ser valorados y tratados oportunamente, para evitar que las lesiones de la piel se conviertan en categorías III y IV, debido a la complejidad y los costes que implican este tipo de lesiones, de acuerdo con la experiencia propia y de otros autores ${ }^{16}$.

Las UPP constituyen un problema general y son altamente costosas en términos de utilización de recursos y efecto perjudicial sobre la calidad de vida. Dentro de la CAH existen diferentes tipos de apósitos que pueden ser usados como apósito primario para las UPP de categorías I o 
II ${ }^{17}$. En este estudio se utilizaron apósitos de poliuretano y un ungüento tópico enzimático, y con ello se observó también una mejoría que supera en mucho a la CT. Éticamente, no se completó la muestra debido a que fue contundente el resultado durante la aplicación de la CAH, y no se pudo negar el beneficio a los pacientes que recibirían CT.

La clínica de heridas del hospital en estudio reportó la atención de 765 pacientes con UPP en el año 2016, lo que representó un gasto promedio anual de 6056214,3 pesos mexicanos (248574,81 euros), para el presupuesto del hospital y de los pacientes; con la implementación de la terapia avanzada, el ahorro hubiera sido un gasto promedio anual de 4338850,5 pesos mexicanos (178086,33 euros).

\section{$\searrow$ CONCLUSIONES}

El uso de la CAH es más coste-efectivo, disminuye los días de cura, el número de consumibles como gasas, agua, guantes, cubre bocas, pańales, etc., y es menor el coste mano-personal. Además, el paciente se encuentra menos tiempo expuesto a infecciones y sufrimiento físico. Una CT es prolongada y puede ser inefectiva en la mayoría de los casos; y finalmente se recurre a la $\mathrm{CAH}$ a pesar del esfuerzo de CT, de tal manera que se termina por invertir en los dos tipos de cura. A pesar de que los apósitos de espuma de poliuretano, ácidos grasos hiperoxigenados y los ungüentos tópicos enzimáticos son más costosos, sus ventajas tecnológicas permiten un menor número de cambios y disminuye los tiempos de cicatrización; por lo tanto, los costes globales disminuyen de manera significativa

\section{Conflicto de intereses}

Los autores declaran no tener ningún conflicto de intereses.

\section{Agradecimiento}

Agradecemos el apoyo de la subdirección de enfermería y la dirección de investigación del Hospital General de México Dr. Eduardo Liceaga.

\section{У BIBLIOGRAFÍA}

1. Silva AJ, Martins PS, Rodrigues A, Rocha AP, Varela J, Gomes LM, et al. Costo económico del tratamiento de las úlceras por presión: una aproximación teórica. Rev Esc Enferm USP. 2013;47(4):967-72.

2. García FFP. Pancorbo HPL, Verdú SJ, Soldevilla AJJ, Rodríguez $\mathrm{PM}$, Gago FM, et al. Eficacia de los productos para el tratamiento de las úlceras por presión: una revisión sistemática con metaanálisis. Gerokomos. 2007;18 (1):36-51.

3. Consenso Internacional. La importancia del tratamiento de heridas eficiente. Wounds International [Internet] Publicado en 2013. 21 páginas. Disponible en: www.woundsinternational. com.

4. Trujillo-Martín, García-Pérez, Serrano-Aguilar. Efectividad, seguridad y coste-efectividad de la terapia por presión negativa tópica para el tratamiento de las heridas crónicas: una revisión sistemática. Rev Med Clin (Barc). 2011;137(7):321-8.

5. Soares PF, Joaquim MM, Dorociak SJ, Crozeta K, Dayane RJ. La efectividad de hidrocoloide versus otros apósitos en la cicatrización de úlceras por presión en adultos y ancianos: revisión sistemática y metaanálisis. Rev Latino-Am Enfermagen. 2014;22(3):511-20.
6. Zárate V. Evaluaciones económicas en salud: Conceptos básicos y clasificación. Rev Med Chile. 2010;138 (2):93-7.

7. Febrer CL, Iglesias GC, Mercadal DJ, Ribera PM. Cómo entender un análisis de coste-efectividad. Piel. [Internet]. 2005 [12 Jul 2018];20(4):172-6

8. Reyes-Rocha BL, Álvarez-Aguirre A, Arciniega-Vega KF, Pozos $A B$. Coste-beneficio de la terapia en ambiente húmedo versus terapia tradicional: el caso de un paciente con úlcera en la extremidad inferior. Gerokomos. 2016;27(2):85-8.

9. García-Fernandez FP, Soldevilla-Agreda JJ, Pancorbo-Hidalgo PL, Verdú Soriano J, López-Casanova P, Rodríguez-Palma M. Clasificación-categorización de las lesiones relacionadas con la dependencia. Serie de Documentos Técnicos GNEAUPP N ${ }^{0} 11$. Grupo Nacional para el Estudio y Asesoramiento en úlceras por Presión y Heridas Crónicas. Logroño. 2014

10. Heyer K, Augustin M, Protz K, Herberger K, Spehr C, Rustenbach $\mathrm{S}$. Effectiveness of advanced versus conventional wound dressings on healing of chronic wounds: systematic review and meta-analysis. Resúmen. Dermatology. 2013;226(2):172-84

11. Barrera AEJ, Pedraza CMC, Pérez JG, Hernández JP, Reyes RJA,
Padilla ZMP Prevalencia de úlceras por presión en un hospital de tercer nivel, en México DF. Gerokomos. 2016;27(4):176-81.

12. Torra-Bou J-E, García-Fernández FP, Pérez-Acevedo G, Sarabia-Lavin R, Paras-Bravo P, Soldevilla-Ágreda JJ, Rodríguez-Palma M, et al. El impacto económico de las lesiones por presión. Revisión bibliográfica integrativa. Gerokomos. 2017;28(2).

13. Soldevilla JJ, Torra i Bou J-E, Posnett J, Verdú SJ, San ML, Mayan SJM. Una aproximación al impacto del coste económico del tratamiento de las úlceras por presión en España. Gerokomos. 2007;18(4):201-10.

14. Restrepo MJC, Escobar CL, Cadavid LM, Muñoz VA. Propuesta de atención al paciente con úlceras por presión (UPP) a través del proceso de atención de enfermería (PAE). Medicina UPB. 2013;32(1):68-78.

15. Nova RJ, Romero DP Pérez HD. Ariza PJ Impacto de coste efectividad en la cura avanzada versus cura tradicional en úlcera venosa. Enferm Dermatol. 2017;11(32):40-4.

16. Vela-Anaya. Magnitud del evento adverso. Ulceras por presión. Rev Enferm Inst Mex Seguro Soc. 2013:21(1):3-8.

17. Fletcher J, Moore Z, Anderson I, Matsuzaki K. Úlceras por presión e hidrocoloides. Wounds Int. 2011;4(2):1-6. 